\title{
LICENCIAS AMBIENTALES: APROXIMACIÓN Y VALORACIÓN DESDE EL ORIGEN DEL CONFLICTO AMBIENTAL*
}

\author{
Claudia Alexandra Munévar Quintero**
}

Presentado: noviembre 22 de 2013 • Aceptado: diciembre 26 de 2013

\section{Resumen}

El siguiente artículo presenta los avances de investigación del proyecto titulado: "Minería, Derechos Humanos y Conflicto: Estado, avances y prospectiva de la explotación minera y su incidencia sobre los Derechos Humanos". En primer lugar, se presenta una contextualización y definición de las licencias ambientales en Colombia. En segundo lugar, se establece una relación entre el origen del conflicto ambiental a partir del otorgamiento de una licencia ambiental. Por último, se analizan los criterios de valoración de las licencias ambientales como normas jurídicas. De igual forma, se presenta una relación sobre la metodología y tipos de investigación del proyecto. Finalmente, se realizan las primeras conclusiones de la fase descriptiva del proyecto de investigación con relación a las licencias ambientales como originadoras de conflictos ambientales.

Palabras clave: licencias ambientales, conflicto ambiental, validez, eficacia y legitimación.

Resultado de avance del proyecto de investigación denominado "Minería, Derechos Humanos y Conflicto: Estado, avances y prospectiva de la explotación minera y su incidencia sobre los Derechos Humanos", adscrito al centro de Investigación Socio-Jurídicas de la Facultad de Ciencias Jurídicas de la Universidad de Manizales.

** Abogada y Magíster en Desarrollo Sostenible y Medio Ambiente de la Universidad de Manizales. Especialista en Derecho Administrativo de la Universidad de Caldas. Candidata a Doctorado en Desarrollo Sostenible de la Universidad de Manizales. Directora del Centro de Investigaciones Socio-Jurídicas de la Facultad de Ciencias Jurídicas de la Universidad de Manizales.

cmunevar@umanizales.edu.co 


\title{
ENVIRONMENTAL LICENSES: APPROACH AND EVALUATION FROM THE ORIGIN OF THE ENVIRONMENTAL CONFLICT
}

\begin{abstract}
This article presents the progress of the research project entitled "Mining, Human Rights and Conflict: State, progress and prospects of mining and its impact on Human Rights". Contextualization and definition of Environmental Licenses in Colombia is presented first. Second, a relationship between the origins of environmental conflict from the granting of an environmental permit is established. Finally, the valuation of environmental licenses and legal standards are analyzed. Similarly, a report on the types of research methodology and the project is presented. Finally, the first findings of the descriptive phase of the research project concerning environmental licenses as originators of environmental conflicts are made.
\end{abstract}

Keywords: Environmental licenses, environmental conflict, validity, effectiveness and legitimacy.

\section{PERMIS D'ENVIRONNEMENT: APPROCHE ET ÉVALUATION DE L'ORIGINE DES CONFLITS DE L'ENVIRONNEMENT}

\section{Résumé}

Ce documentprésente l' étatd'avancement du projet de recherche intitulé «Mines, droits de l'homme et les conflits: Etat, les progrès et les perspectives de l'exploitationminière et de son impact sur les droits de l'homme» Contextualisation et la définition des licencesenvironnementales en Colombiesontprésentés en premier. Deuxièmement, une relation entre l'origine du conflit de l'environnement de l'octroid'unpermisenvironnementalestétabli. Enfin, l'évaluation des licencesenvironnementales et les normes juridiquessontanalysés. De même, un rapport sur les types de méthodologie de la recherche et le projetestprésenté. Enfin, les premiersrésultats de la phasedescriptive 
du projet de rechercheconcernant les permisenvironnementauxsont des initiateurs de conflitsenvironnementauxsonteffectués.

Mots-clés: permisd'environnement, les conflits de l'environnement, la validité, l'efficacité et la légitimité.

\section{LICENZE AMBIENTALI: APPROSSIMAZIONE E VALUTAZIONE DALL'ORIGINE DEL CONFLITTO AMBIENTALE}

\section{Riassunto}

Il seguente articolo presenta i progressi del progetto di ricerca che ha per titolo titolo: "Mineraria, Diritti Umani e Conflitto: Stato, progresso e prospettiva del sfruttamento miniero e il suo impatto sui Diritti Umani". Prima di tutto, viene presenta una contestualizzazione e definizione delle licenze ambientali in Colombia. In secondo luogo, si è stabilita una relazione tra l'origine del conflitto ambientale dalla concessione di una licenza ambientale. Infine, si analizzano i criteri di valutazione delle licenze ambientali come norme giuridiche. Analogamente, viene presentata una relazione sulla metodologia e tipi di ricerca del progetto. Finalmente, sono svolte le prime conclusioni della fase descrittiva del progetto di ricerca con relazione alle licenze ambientali come originatore di conflitti ambientali.

Parole chiave: licenze ambientali, conflitti ambientali, validità, efficacia e legittimità.

\section{LICENÇAS AMBIENTAIS: APROXIMAÇÃO E VALORAÇÃO DESDE A ORIGEM DO CONFLITO AMBIENTAL}

\section{Resumo}

O seguinte artigo apresenta os avanços de pesquisa do projeto intitulado: "Minaria, Direitos Humanos e Conflito: Estado, avanços e prospectiva da exploração mineira e sua incidência sobre os Direitos 
Humanos". No primeiro lugar, apresenta-se uma contextualização e definição das Licenças Ambientais na Colômbia. No segundo lugar, é estabelecida uma relação entre a origem do conflito ambiental a partir da conferição de uma licença ambiental. Finalmente, são analisados os critérios de valoração das licenças ambientais como normas jurídicas. Do mesmo jeito, é apresentada uma relação sobre a metodologia e tipos de pesquisa do projeto. Afinal, são realizadas as primeiras conclusões da fase descritiva do projeto de pesquisa com relação às Licenças Ambientais como originadoras de conflitos ambientais.

Palavras chave: licenças ambientais, conflito ambiental, validez, eficácia e legitimação. 


\section{INTRODUCCIÓN}

Dentro de las diferentes formas de comprender el Derecho se han desarrollado diferentes escuelas, posturas y teorías en torno a éste. Sin duda, una de las preguntas y cuestionamientos más problemáticos está relacionado con los conceptos de validez, eficacia y legitimidad del derecho, los cuales se confrontan a la luz de perspectivas fácticas y axiológicas que replantean el verdadero alcance y finalidad de la norma. Estas temáticas, que han ocupado las reflexiones teóricas y jurídicas, trascienden de una mera reflexión especulativa en el campo de la academia a una mirada práctica y fáctica de las necesidades sociales, que esperan y exigen del Derecho una respuesta.

Una de estas necesidades sociales es la denominada crisis ambiental, la cual ha sido catalogada como una de las más grandes de esta época. Esta crisis, por sus especiales características, ha concentrado la atención de la comunidad internacional y de diferentes ciencias, disciplinas y áreas del conocimiento, dentro de las cuales el Derecho ha empezado a intervenir a través de su normatividad ambiental, que a pesar de ser reciente, sí se ha caracterizado por ser una de las áreas de mayor evolución en su estudio y producción legislativa y administrativa.

No obstante, y a pesar de reconocerse no sólo una producción legislativa sino una verdadera conformación de una nueva rama del Derecho, como lo es el Derecho Ambiental, las problemáticas y conflictos ambientales parecieran desbordar este alcance y cuestionar la legitimidad de este Derecho.

El presente artículo refleja los avances de investigación del proyecto titulado: "Minería, derechos humanos y conflicto: Estado, avances y prospectiva de la explotación minera y su incidencia sobre los derechos humanos". Este proyecto de investigación corresponde a una investigación socio-jurídica ${ }^{1}$ y cualitativa, la cual plantea el siguiente objetivo general: analizar la incidencia de la explotación minera en la vulneración de los derechos humanos en Colombia.

\footnotetext{
"La investigación socio-jurídica estudia la realidad social, porque su objeto es actuar sobre los comportamientos sociales, pero con miras a regularlos para lograr los fines políticos que se propone un Estado en un momento histórico determinado. Ella no se queda en el conocimiento de los fenómenos sociales, sino que después de conocerlos, los trasciende y los valora con relación a dichos fines, para definir de qué manera los debe modificar para alcanzar las metas buscadas" (Giraldo et al., 2010, p. 3).
} 
Este proyecto está siendo desarrollado en cuatro fases: fase descriptiva, analítica, comprensiva y prospectiva. Cada una de ellas corresponde a un tipo y momento de investigación diferente. Los avances presentados en este artículo conciernen a la primera fase, correspondiente a un tipo de investigación descriptiva $^{2}$ y al siguiente objetivo específico de investigación: describir, a partir de casos, el estado actual de la explotación minera en Colombia en torno a la normatividad minero ambiental y su influencia sobre los derechos humanos.

El presente artículo se orientará al análisis del origen de los conflictos ambientales a partir del otorgamiento y expedición de las licencias ambientales. En este sentido se genera una confrontación desde el marco de la legalidad de las licencias como causantes del conflicto ambiental, surgiendo cuestionamientos relacionados con la finalidad de la norma, tales como la justicia, la equidad, la igualdad y otros aspectos axiológicos.

\section{UNA APROXIMACIÓN AL CONCEPTO DE LICENCIAS AMBIENTALES}

Las licencias ambientales constituyen el instrumento por medio del cual se hacen efectivas las disposiciones de los instrumentos jurídicos internacionales, la Constitución Política y la Ley para la protección del medio ambiente y la prevención y mitigación del impacto ambiental. En un primer sentido, dentro del marco del Derecho Ambiental Internacional se ha establecido el principio de prevención, el cual, en palabras de Rojas (2004, p.172) "genera obligaciones específicas y [...] una serie de comportamientos implícitos en el concepto de prevención". De esta forma las licencias ambientales constituyen el instrumento para la prevención y mitigación del impacto ambiental.

En el marco de la Constitución Política de 1991, el Artículo 80³, establece que es responsabilidad del Estado planificar el manejo de los recursos

2 "La importancia de la investigación descriptiva radica, entre otras cosas, en que ella, junto con la investigación exploratoria, constituye la base y el punto de partida para los tipos de investigación de mayor profundidad. En la medida que existen descripciones precisas de los eventos, es más sencillo avanzar a niveles de mayor complejidad [...]. Las investigaciones de diferente tipo necesitan pasar por el estadio descriptivo para obtener la información que se necesita en los siguientes estadios: ya sea que el propio investigador realice la descripción, o que ésta haya sido hecha por otros investigadores" (Hurtado de Barrera, 2010, p. 414).

3 "El Estado planificará el manejo y aprovechamiento de los recursos naturales, para garantizar su desarrollo sostenible, su conservación, restauración o sustitución. Además, deberá prevenir y controlar los factores de deterioro ambiental, imponer las sanciones legales y exigir la reaparición de los daños causados". 
naturales para garantizar su desarrollo sostenible; así, las licencias ambientales son el mecanismo por medio del cual se hace efectiva ésta disposición constitucional, por medio de un acto administrativo, que en palabras de Rodríguez:

La autorización que otorga la institución ambiental competente, conocida como licencia ambiental, es en esencia un acto administrativo que se debe reconocer como el medio directo por excelencia, para el cumplimiento de los fines y propósitos estatales en materia de protección y conservación de los recursos naturales y del ambiente (Rodríguez et al., 2012, p. 51).

En este sentido, con un antecedente internacional y con una fuente constitucional, en Colombia se desarrolla la licencia ambiental a partir de la Ley 99 de 1993, definida en el Artículo 50 como:

Se entiende por Licencia Ambiental la autorización que otorga la autoridad ambiental competente para la ejecución de una obra o actividad, sujeta al cumplimiento por el beneficiario de la licencia de los requisitos que la misma establezca en relación con la prevención, mitigación, corrección, compensación y manejo de los efectos ambientales de la obra o actividad autorizada.

La licencia ambiental como mecanismo administrativo no sólo adquiere una connotación ambiental sino económica y social. En primer lugar, en cumplimiento del principio de prevención, adopta mecanismos como el Estudio de Impacto Ambiental, el cual, en palabras de Medellín (1997, p. 15) "mide el grado de vulneración del Medio Ambiente cuando se va a emprender un proyecto determinado. Se dirige a sugerir las medidas más apropiadas para efectos de prevenir, mitigar o controlar la consecuencia del impacto en determinada actividad". Así, los instrumentos de la licencia ambiental tienen como finalidad la protección de los recursos naturales en la puesta en marcha de una obra, actividad o proyecto que modifique el paisaje o que genere graves daños o impactos significativos al medio ambiente. 
Respecto al sentido social, la licencia ambiental consagra modos y procedimientos de participación ciudadana, contemplados en la Ley 99 de 1993, tales como: el derecho a intervenir en los procedimientos administrativos ambientales ${ }^{4}$, el trámite de las peticiones de intervención ${ }^{5}$, las audiencias públicas administrativas sobre decisiones ambientales en trámite ${ }^{6}$, la acción de nuli$\mathrm{dad}^{7}$, el derecho de petición ${ }^{8}$ y la consulta previa ${ }^{9}$. Si se llegaran a incumplir generan al beneficiario medidas sancionatorias, revocación de la licencia, suspensión o demolición de la obra e imposición de sanciones, entre otras.

4 ARTÍCULO 69. DEL DERECHO A INTERVENIR EN LOS PROCEDIMIENTOS ADMINISTRATIVOS AMBIENTALES.Cualquier persona natural o jurídica, pública o privada, sin necesidad de demostrar interés jurídico alguno, podrá intervenir en las actuaciones administrativas iniciadas para la expedición, modificación o cancelación de permisos o licencias de actividades que afecten o puedan afectar el medio ambiente o para la imposición o revocación de sanciones por el incumplimiento de las normas y regulaciones ambientales.

5 ARTÍCULO 70. DEL TRÁMITE DE LAS PETICIONES DE INTERVENCIÓN. La entidad administrativa competente al recibir una petición para iniciar una actuación administrativa ambiental o al comenzarla de oficio dictará un acto de iniciación de trámite que notificará y publicará en los términos de los artículos 14 y 15 el Código Contencioso Administrativo y tendrá como interesado a cualquier persona que así lo manifieste con su correspondiente identificación y dirección domiciliaria. Para efectos de la publicación a que se refiere el presente artículo toda entidad perteneciente al Sistema Nacional Ambiental publicará un Boletín con la periodicidad requerida que se enviará por correo a quien lo solicite.

6 ARTÍCULO 72. DE LAS AUDIENCIAS PÚBLICAS ADMINISTRATIVAS SOBRE DECISIONES AMBIENTALES EN TRÁMITE.El Procurador General de la Nación o el Delegado para Asuntos Ambientales, el Defensor del Pueblo, el Ministro del Medio Ambiente, las demás autoridades ambientales, los gobernadores, los alcaldes o por lo menos cien (100) personas o tres (3) entidades sin ánimo de lucro, cuando se desarrolle o pretenda desarrollarse una obra o actividad que pueda causar impacto al medio ambiente o a los recursos naturales renovables, y para la cual se exija permiso o licencia ambiental conforme a la ley o a los reglamentos, podrán solicitar la realización de una audiencia pública que se celebrará ante la autoridad competente para el otorgamiento del permiso o la licencia ambiental respectiva.

$7 \quad$ ARTÍCULO 73. DE LA CONDUCENCIA DE LA ACCIÓN DE NULIDAD.La acción de nulidad procede contra los actos administrativos mediante los cuales se expide, modifica o cancela un permiso, autorización, concesión o Licencia Ambiental de una actividad que afecte o pueda afectar el medio ambiente.

8 ARTÍCULO 74. DEL DERECHO DE PETICIÓN DE INFORMACIONES. Toda persona natural o jurídica tiene derecho a formular directamente petición de información en relación con los elementos susceptibles de producir contaminación y los peligros que el uso de dichos elementos pueda ocasionar a la salud humana de conformidad con el Artículo 16 de la Ley 23 de 1973. Dicha petición debe ser respondida en diez (10) días hábiles. Además, toda persona podrá invocar su derecho a ser informada sobre el monto y utilización de los recursos financieros, que están destinados a la preservación del medio ambiente.

9 ARTÍ́CULO 76. DE LAS COMUNIDADES INDÍGENAS Y NEGRAS. La explotación de los recursos naturales deberá hacerse sin desmedro de la integridad cultural, social y económica de las comunidades indígenas y de las negras tradicionales de acuerdo con la Ley 70 de 1993 y el Artículo 330 de la Constitución Nacional, y las decisiones sobre la materia se tomarán, previa consulta a los representantes de tales comunidades. 
Por último, la licencia ambiental, en cumplimiento al principio de Desarrollo Sostenible, tiene una connotación económica toda vez que uno de sus criterios lo constituye la planificación del medio ambiente con el fin de lograr la conservación del mismo, pero a su vez, logrando el desarrollo económico a través del aprovechamiento de los recursos naturales.

En este sentido, es la licencia ambiental el instrumento establecido por el legislador para el otorgamiento de permisos por parte de la autoridad ambiental y para la ejecución de proyectos que impacten los recursos naturales. Así, es considerado como el mecanismo idóneo para la protección del medio ambiente ante el impacto que pueda generar determinada obra o proyecto.

Ahora bien, la realidad social y los actuales conflictos ambientales han cuestionado la eficacia de este instrumento, toda vez que, a partir del otorgamiento de licencias ambientales se han desatado importantes conflictos socio-ambientales en Colombia, como por ejemplo, el caso de la comunidad indígena U'wa y el del proyecto Mandé Norte, ambos casos relacionados con la explotación minera.

El primero de ellos es el mencionado en la Sentencia SU-039 de 1997 de la Corte Constitucional. La comunidad indígena U'wa, localizada en los departamentos de Boyacá, Norte de Santander, Santander, Arauca y Casanare, considera vulnerados sus derechos fundamentales a partir de la expedición de la Resolución 110 de febrero 3 de 1995 del Ministerio del Medio Ambiente, la cual otorgó licencia ambiental a la Occidental de Colombia Inc. El segundo caso es la explotación minera adelantada por la empresa Muriel Mining Corporation en el marco del proyecto Mandé Norte en los departamentos de Antioquia y Chocó, donde se encuentra ubicado un resguardo indígena embera de Uradá Jiguamiandó, además de otras comunidades afrodescendientes. La licencia ambiental otorgada por la Autoridad Ambiental al contrato de concesión minera permitió que la Corte Constitucional en Sentencia T-769 de 2009, se pronunciara ordenando al entonces Ministro de Ambiente, Vivienda y Desarrollo Territorial que "se emitan licencias ambientales para la ejecución de proyectos de exploración y explotación que afecten la biodiversidad".

Casos como estos evidencian que el sector minero en Colombia está considerado como una de las grandes locomotoras de desarrollo, según el Plan Nacional de Desarrollo 2011-2014; así lo confirman Estupiñán y Polanía: 
El Plan Nacional de Desarrollo "Prosperidad para todos", hace énfasis en las locomotoras del desarrollo para señalar aquellos sectores que avanzan o deberían avanzar más rápido que el resto de la economía. Este es el caso del sector de la minería. Las cifras de inversión, productividad y empleo del sector permiten pensar que tendrá un crecimiento mayor al de la economía en conjunto (2011, p. 44).

Sin embargo, esta prospectiva de desarrollo en Colombia que ha estimulado la inversión extranjera y a su vez han generado diferentes conflictos ambientales a partir de la expedición de licencias ambientales, evidencia el cuestionamiento de la legitimidad de la norma jurídica y como, a partir de ella, se crean diferentes conflictos ambientales.

\section{LICENCIAS AMBIENTALES: ORIGEN DEL CONFLICTO AMBIENTAL}

El concepto conflicto generalmente está asociado a situaciones litigiosas, controversiales o de problemas. En el lenguaje jurídico existen diferentes definiciones que así lo establecen. Para Goldstein (2010, p. 155) el conflicto es definido como: "oposición y choque de intereses entre distintos sujetos de derechos". De igual forma, para Ossorio (1997, p.152) la palabra conflicto hace referencia a "choque o colisión de derechos o pretensiones". Para Madrid-Malo (2005, p. 69) el conflicto es: "Una situación en la cual se enfrentan en un plano de oposición dos o más partes que por cualquier causa han llegado a ser incompatibles".

Estas definiciones logran asociar el conflicto con problemas o situaciones negativas, sin embargo, Felstiner, Abel y Sarat (2001, p. 41) afirman que "los conflictos no son cosas: son construcciones sociales". Ahora bien, asociar al conflicto con una construcción social implica, necesariamente, romper con un paradigma clásico del concepto y entrar a comprenderlo desde una perspectiva más crítica, interpretativa y por supuesto, social.

Para entrar en una discusión más profunda sobre el alcance de este concepto, es necesario entender y establecer qué es una construcción social. Para esta definición, Felstiner, Abel y Sarat establecen esa construcción social como un proceso de trasformación que inicia desde el origen del conflicto, pasa a una fase de percepción del mismo, posteriormente a una etapa de acusación y finalmente llega a la reclamación, aquella que los autores definen como la materialización del acceso a la justicia: "El hecho de tener en cuenta las etapas de reconocimiento, acusación y reclamación nos permite ver con 
ojos más críticos los recientes esfuerzos por mejorar el acceso a la justicia" (2001, p.46). Sin embargo, para este trabajo la trasformación no se agota con la reclamación, toda vez que esta construcción social y esa trasformación del conflicto se resuelve en una respuesta por parte de esa administración de justicia, más aún cuando se habla de conflictos ambientales.

Avanzando un poco hacia la re-conceptualización del concepto de conflicto, específicamente del conflicto ambiental, es fundamental hacer una relación a lo que produce su origen. Generalmente, y acorde a la definición de Madrid-Malo (2005, p. 69), "se llama conflicto al enfrentamiento entre normas, derechos, intereses, jurisdicciones o competencias", no es problemático asociar un conflicto ambiental en el plano de la ilegalidad, por ejemplo, un conflicto ambiental generado por un daño producido a raíz de una explotación ilícita de un hidrocarburo, por la explotación ilegal de unos recursos, por sobrepasar los límites de contaminación permitida, por iniciar un proyecto sin los respectivos permisos y licencias, entre otros.

Sin embargo, a la hora de analizar un conflicto ambiental originado por el otorgamiento de una licencia ambiental, se replantea la idea de que el origen del conflicto se dé en el plano de la ilegalidad, toda vez que la licencia ambiental goza de esta legitimidad y cuenta con plena validez. Como ejemplo se puede mencionar el otorgamiento de una licencia ambiental para la explotación de determinados recursos o para la elaboración de un proyecto. Esta licencia ambiental, que es otorgada por una autoridad competente, puede originar un conflicto de orden social y ambiental, ya que involucra derechos de individuos, comunidades y jurisdicciones, y además, impactos significativos al medio ambiente.

En este sentido, vale la pena cuestionar los criterios de validez con los que se asume la legitimación de las licencias ambientales, es decir, si éstas deben ser abordadas desde el plano netamente normativo o social y si desde su expedición y otorgamiento se deben valorar criterios de justicia.

\section{LICENCIAS AMBIENTALES Y SU LEGITIMACIÓN COMO NORMAS JURÍDICAS}

La legitimación, validez, eficacia, legalidad y eficiencia de la norma han sido conceptos discutidos por diferentes teóricos, los cuales determinan la comprensión de la norma pero varían según la escuela o teoría jurídica que los definen. Por ejemplo, para el positivismo expuesto por Kelsen 
(1952, p. 35) la legitimidad de la norma está determinada por la legalidad; en este sentido, lo legal es legítimo: "El derecho tiene la particularidad de que regula su propia creación y aplicación". Como concluye Giraldo et al. (2010, p. 44), "la ley es además la fuente que legitima su propia creación".

Comprendiendo la legitimación de la norma como la otorgada por el legislador o la autoridad competente que la expide, su legitimidad no depende del contenido ideológico ni axiológico de la misma. Para Kelsen el derecho debe ser analizado desde su estructura:

Estas tendencias ideológicas, cuyos designios y efectos políticos son evidentes dominan todavía la Ciencia Actual, aún después de la aparente superación de la teoría del Derecho Natural. Contra ellas se dirige la Teoría pura del Derecho. Quiere ésta exponer el Derecho tal cual es, sin legitimarlo por justo o descalificarlo por injusto; pregunta por el Derecho real y posible, no por el Derecho justo. [...] concebir al Derecho Positivo con arreglo a su esencia y a comprenderlo por un análisis de su estructura (1977, p. 43).

Sin embargo, esa valoración del Derecho por lo justo o lo injusto se contrapone a las corrientes iusnaturalistas, las cuales, según Giraldo et al., establecen:

La razón por la cual una norma debe ser obedecida radica en el carácter imperativo del principio universal en que se funda. En las concepciones del iusnaturalismo teológico ello es claro, por cuando siendo el derecho positivo expresión del orden divino, el carácter obligatorio de aquél depende de éste. Igual fundamento tienen las concepciones del iusnaturalismo racionalista, pues el derecho positivo no puede ser más que una expresión de un orden natural [...] (2010, p. 35).

En este sentido se puede afirmar que el Derecho tiene diferentes opciones o posiciones de valoración: desde las reglas o desde la moral. Sin embargo, la dimensión social del Derecho, plantea otra perspectiva de la noción de validez; esta concepción es la de la Sociología Jurídica, la cual, según Giraldo et al. (2010, p. 92) plantea: "En la concepción Sociológica se requiere además una validez material. Por esa razón él está integrado por un conjunto de estatutos normativos expedidos expresamente para resolver los conflictos que ofrece una comunidad en un momento histórico determinado".

Sin embargo, a pesar de la presentación de las posturas teóricas y epistemológicas de cada una de estas corrientes de la teoría del Derecho, se puede observar que "existe una relación problemática entre las dimensiones 
epistemológicas de la legitimidad, la validez y la eficacia" (Hernández et al., 2010, p. 51), y al exponer las corrientes de Weber, Kelsen y Hart, los mismos autores concluyen: "Hay entonces una confusión entre legitimidad, validez y eficacia en la enunciación de esa norma fundamental. [...] Una normativa que funda la validez, y una fáctica que funda la eficacia del sistema, lo que motiva a la confusión ontoepistemológica" (2010, pp. 52-53).

De esta manera se ha realizado una aproximación teórica de la licencias ambientales respecto a su valoración desde su "eficacia", cuyo alcance gira alrededor del acatamiento por parte de la sociedad; su "validez", entendiendo lo válido como lo ordenado, determinado por la autoridad competente que expidió ésta norma jurídica; y, finalmente, por su "legitimidad", concepto que es comprendido según la teoría jurídica que lo desarrolle y que, para efectos de la investigación adelantada, es el criterio de valoración que se cuestiona en cuanto a la vulneración de los derechos humanos a raíz de un conflicto ambiental originado a partir del otorgamiento de la licencia ambiental.

\section{CONCLUSIONES}

El conflicto ambiental, asociado generalmente a la confrontación de normas o quebrantamiento de las mismas, se asimila, desde su origen, en el plano de la ilegalidad. No obstante, cuando se entienden las licencias ambientales como los permisos otorgados por la autoridad competente para la ejecución de una obra o proyecto, y que por su naturaleza jurídica son denominadas como actos administrativos, esto es, norma jurídica, no cabe duda que sean el instrumento legal e idóneo para la planificación de los recursos naturales por parte del Estado.

Sin embargo, la actual crisis ambiental y los recientes conflictos ambientales han llevado a la confrontación de las licencias ambientales, como normas jurídicas, por ser las causantes y originarias de los conflictos ambientales, y por ende, a la vulneración de los derechos humanos. Esta última idea se presenta como una categoría emergente en los avances del proyecto de investigación adelantado, lo cual contribuye a plantear el interrogante de la legitimidad de las licencias ambientales, no desde su criterio de validez sino desde su criterio de eficacia y de correspondencia con lo justo.

Concebir el origen de un conflicto desde el plano de la legalidad ayuda al replanteamiento, no sólo del concepto del conflicto sino a la legitimidad y concepción de la norma jurídica. Ésta última, como una norma 
válida, toda vez que ha sido expedida por una autoridad competente, debe preguntarse por criterios de eficacia y de justicia. La actual crisis ambiental y los recientes conflictos demandan del Derecho el cuestionamiento de lo justo, de lo injusto, de su aplicación en la sociedad y de la eficacia de la norma en la misma. No basta con que una licencia ambiental sea expedida por una autoridad competente para el cumplimiento de un fin determinado en la ley que las regula; es preciso y determinante que las mismas obedezcan a criterios valorativos y necesidades sociales. Toda vez que, en el desconocimiento de los valores y de la realidad, las licencias ambientales serán las causantes de los conflictos ambientales y por ende de la vulneración de los Derechos Humanos.

\section{REFERENCIAS BIBLIOGRÁFICAS}

Constitución Política de Colombia (2004). Colombia: Editorial Leyer. Estupiñán, F. y O. Polanía (2011). "Las locomotoras del desarrollo: minas, energía e innovación". Revista de Ingeniería. Bogotá: Universidad de los Andes.

Felstiner, W. L. F; L. A. Richard y A. Sarat (2001). "Origen y trasformación de los conflictos: reconocimiento, acusación, reclamación”. En: M. García Villegas (ed.). Sociología Jurídica. Bogotá: Universidad Nacional de Colombia.

Giraldo, J.; M. Giraldo y A. Giraldo (2010). Metodología y técnica de la investigación socio-jurídica (4. ${ }^{\mathrm{a}}$ ed.). Bogotá: Librería Ediciones del Profesional.

Goldstein, M. (2010). Diccionario jurídico. Bogotá: Editorial Cultural Internacional.

Hernández, R.; P. Baptista y C. Fernández (2010). Metodología de la investigación (5. ${ }^{\mathrm{a}}$ ed.). México: Mc Graw Hill.

Hurtado de Barrera, J. (2006). El proyecto de investigación: metodología de la investigación holística (4. ${ }^{a}$ ed.). Bogotá: Quirón Ediciones.

(2010). Metodología de la investigación: guía para la comprensión holística de la ciencia (4. ${ }^{a}$ ed.). Bogotá: Quirón Ediciones.

Kelsen, H. (1952). Problemas escogidos de la Teoría Pura del Derecho. Buenos Aires: Editorial Guillermo Kraft. (1977). Teoría Pura del Derecho (15. ${ }^{\mathrm{a}}$ ed.). Buenos Aires: Editorial Universitaria. 
Madrid-Malo, M. (2005).Diccionario de la Constitución Política de Colombia (3. ${ }^{\mathrm{a}}$ ed.). Bogotá: Librería Ediciones del Profesional.

Medellín, C. (1997). "Instrumentos internacionales y legales de protección al medio ambiente en Colombia". En: Memorias seminario taller, Fortalecimiento de los mecanismos judiciales de protección del medio ambiente (2. ${ }^{a}$ ed.). Bogotá: Ediciones Beatriz Londoño Toro.

Ossorio, M. (1997).Diccionario jurídico. Uruguay: Editorial Obra Grande.

Rodríguez, G. A.; A. Gómez y J. C. Monroy (2012).Las licencias ambientales en Colombia. Bogotá: Editorial Ibáñez.

Rojas, C. M. (2004). Evolución de las características y de los principios del derecho internacional ambiental y su aplicación en Colombia. Bogotá: Departamento de Publicaciones Universidad Externado de Colombia. 
